\title{
Significance of Toll-like Receptors Expression in Tumor Growth and Spreading: A Short Review
}

\author{
Jan Zeromski • Iwona Mozer-Lisewska • \\ Mariusz Kaczmarek
}

Received: 31 December 2007 / Accepted: 28 January 2008 / Published online: 29 February 2008

(C) Springer Science + Business Media B.V. 2008

\begin{abstract}
Toll-like receptors (TLRs) are considered now as crucial sensors of innate immunity. Their role in the recognition of pathogens and the initiation of adaptive immune responses against them is well known. However, in last years TLRs have been identified on several tumor cells, including human malignancies. Their expression in cancer was found to be twofold: either promoting or inhibiting tumor progression. It was also demonstrated that several TLRs agonists, either natural or synthetic ones, may have beneficial effect on tumor-mediated disease, leading to potentiation of immune response to tumor-associated antigens. TLR-agonist linked tumor immunotherapy is still in nascent state, but growing rapidly, also in the area of common human malignancies. To date, the most promising and the most frequently studied interaction in tumor immunotherapy trials seems to be TLR9 and its synthetic agonists.
\end{abstract}

Keywords Toll-like receptors · Pathogen-associated molecular patterns $\cdot$ Innate immunity $\cdot$ Signaling .

Tumor cells $\cdot$ Agonists

$\begin{array}{ll}\text { Abbreviations } \\ \text { TLRs } & \text { Toll-like receptors } \\ \text { PRRs } & \text { pattern recognition receptors } \\ \text { TIR } & \text { Toll/interleukin-1 receptor domain } \\ \text { PAMPs } & \text { pathogen associated molecular patterns }\end{array}$

J. Zeromski $(\bowtie) \cdot$ M. Kaczmarek

Clinical Immunology, Medical University,

Rokietnicka 5D,

Poznan 60-806, Poland

e-mail: jzeromski@ump.edu.pl

I. Mozer-Lisewska

Infectious Diseases and Child Neurology, Medical University,

Szpitalna 27/33,

Poznan 60-572, Poland
CpG-ODN CpG-oligonucleotides

MyD88 myeloid differentiation factor 88

IRAK interleukin-1 receptor associated kinase

MAPK mitogen-activated protein kinase

NF- $k B \quad$ nuclear factor $k B$

AP-1 activated protein-1

HSP heat shock proteins

HNSCC head and neck squamous cell carcinoma

TRIF TIR domain-containing adaptor protein inducing interferon- $\beta$

IRF3 interferon regulatory factor 3

SIGIRR single immunoglobulin IL-1-related protein

Tollip Toll-interacting protein

TNF tumor necrosis factor

TRAF TNF receptor-associated factor

TRAIL TNF-related apoptosis inducing ligand

TGF $\beta \quad$ transforming growth factor- $\beta$

LPS lipopolysaccharide

MALP-2 macrophage activating lipopeptide-2

RAC1 ras-related C3 botulinum toxin substrate 1 (rho family, small GTP binding protein Rac1)

PI3K phosphoinositide-3-kinase

AKT v-akt murine thymoma viral oncogene homolog 1

TIRAP Toll-interleukin 1 receptor (TIR) domain containing adaptor protein

FADD Fas (TNFRSF6)-associated via death domain

TAK cyclin-dependent kinase 9

TAB mitogen-activated protein kinase kinase kinase 7 interacting protein

IKK inhibitor of kappa light polypeptide gene enhancer in B-cells

TRAM Toll-like receptor adaptor molecule 2

RIP ralA-binding protein 


\section{Introduction}

Toll-like receptors (TLRs) a group out of evolutionally conserved family of pattern recognition receptors (PRRs) have gained great interest in a large community of life science researchers $[1,2]$. TLRs recognize mainly products of microbial origin, known as pathogen-associated molecular patterns (PAMPs). The latter include a large variety of various organic compounds such as lipopolysaccharides (LPS) from Gram-negative bacteria, viral single and double-stranded RNAs, various sugars, unmethylated $\mathrm{CpG}$ oligonucleotides (CpG-ODN) and others. To date, 10 members of TLR family, (designed from TLR1 to TLR10) have been identified in humans, reflecting the reactivity with particular PAMPs [3]. In functional terms, TLRs, following dimerization, signal through the cytoplasmic Toll/interleukin-1 receptor domain (TIR). Several other adaptor molecules and enzymes participate in the signaling, including MD-2, MyD88, IL-1 receptor-associate kinases
(IRAK-4 and IRAK-1), TNF-receptor associated factor (TRAF), mitogen-activated protein kinase (MAPK) and others. Out of them MyD88 (myeloid differentiation primary-response protein 88 ) adaptor protein seems to be the most important, because all TLRs with the exception of TLR3, require MyD88 pathway for the initiation of synthesis of proinflammatory cytokines. Besides, MyD88 possess N-terminal death domain enabling it to interact with IL-1 receptor-associated kinase (IRAK). TLR3 uses MyD88-independent/TRIF-dependent pathway to induce IRF3, being critical for the induction of antiviral immunity. The net effect of signaling is the activation of transcription factors such as NF- $\mathrm{KB}$ and AP-1. Their entrance to the cell nucleus result in the transcription of several genes encoding a variety of biologically relevant macromolecules such as proinflammatory cytokines, chemokines, growth factors etc. Simplified biochemical events in cell interior happening after various TLRs signaling are shown in Fig. 1. Precise biochemical sequence of incidents accompanying TLR

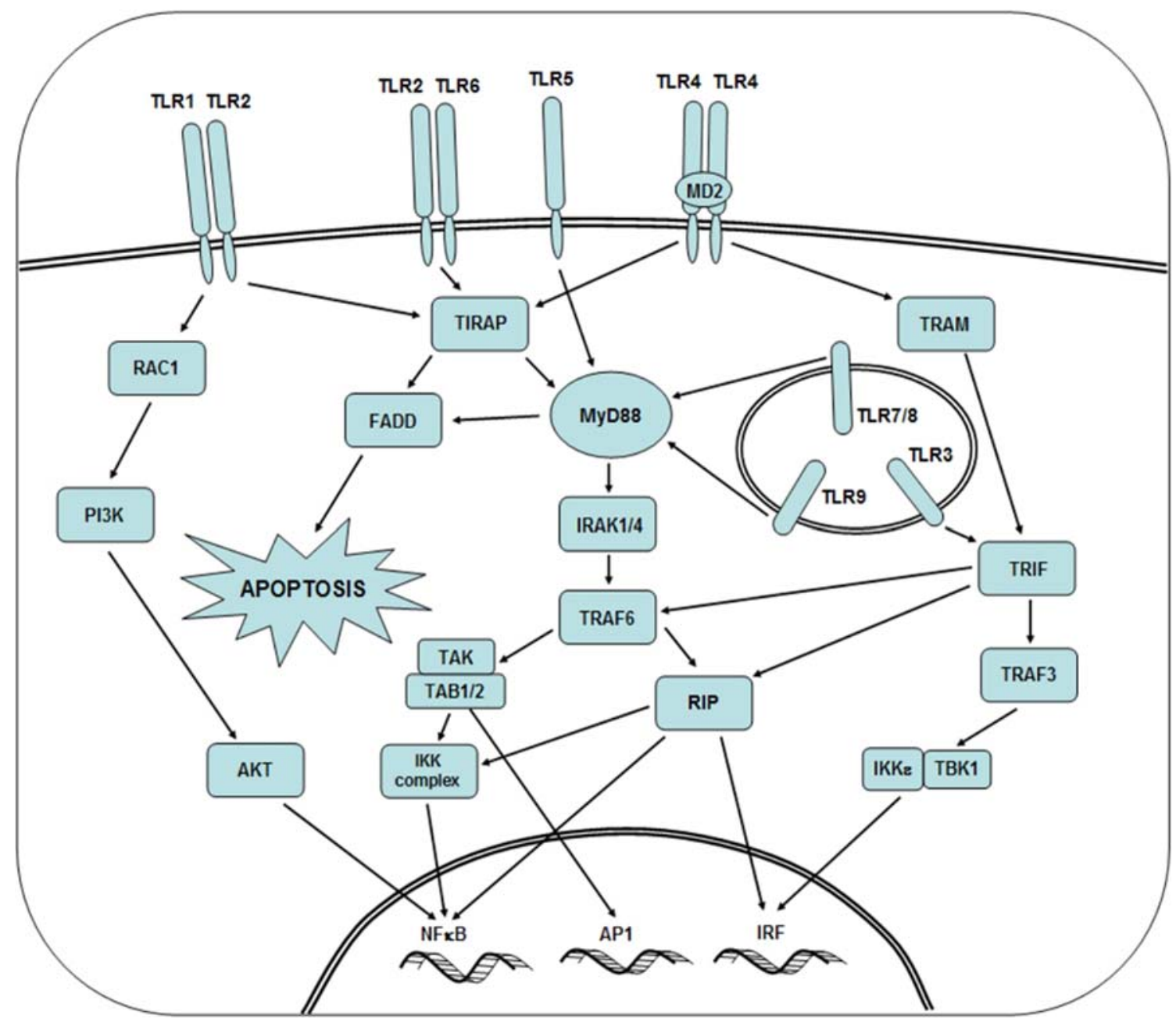

Fig. 1 Signal transduction pathways of Toll-like receptors. TLRs are both, extracellular and intracellular. Individual TLRs have various pathways of signal transduction, either $\mathrm{MyD}^{+} 8^{+}$or MyD88 ${ }^{-}$(TLR3 only and partly TLR1/TLR2). Binding of ligand by TLR results in the activation of cascade of kinases, leading to the entry of transcription factors, such as NF-kB, AP1 and IRF to cell nucleus 
stimulation, various ways of signaling used by particular TLRs are outside the scope of the present review and may be found elsewhere [4]. The opinion is generally held that TLR signaling constitutes a key component of the innate immune response to microbial infection $[5,6]$.

TLRs are expressed in both, lymphoid and non-lymphoid tissues, but expression in the former predominates. Practically all cells of the immune system express TLRs, but the pattern of expression varies with the cell type. Stimuli able to induce activation of TLRs are heterogenous and include, apart from microbial compounds, other agents such as injury induced by excessive heat [7], various chemicals, ionizing radiation etc. There is an evidence that in addition to their function as sensors of foreign PAMPs, TLRs can also mediate responses to endogenous stimuli. The latter comprise various factors such as heat shock proteins (HSP), necrotic debris, fibrinogen, degraded fibronectin fragments [8] and other cell and/or body constituents [9].

Apart from positive stimuli of TLR system, both exogenous and endogenous ones, the system is also under negative regulation, preventing excessive inflammatory responses. There are several agents involved, but good example of them is single immunoglobulin IL-1-related protein (SIGIRR) expressed on epithelial cells of liver, kidney, intestine but also on dendritic cells [10]. SIGIRR is able to inhibit MyD88-related pathway and NF- $\mathrm{KB}$ activation. Several other negative regulators have been described, including extracellular ones such as soluble decoy TLRs, transmembrane (SIGIRR) and intracellular (Toll-interacting protein-Tollip). More data about negative regulation of TLRs are available in recently published excellent review article of Lang and Mansell [11].

\section{TLR Expression on Tumor Cells and their Putative Function}

There is an accumulating and steadily growing evidence that cells of several human malignancies express single or more commonly multiple TLRs. Significance of this phenomenon is far from clear, There are, however several lines of evidence suggesting biological impact of TLRs expression on tumor cell growth and survival. For example, Hassan et al. [12] have shown that cells of human neuroblastoma express intracellular form of TLR4. Moreover, these cells show expression of factors required for LPS response such as CD14, MyD88 and MD2, what suggests, that TLR4 expression is functional.

He et al. [13] demonstrated negative function of TLR4 expression in human lung cancer cell lines. Ligation of LPS agonist by the above mentioned cells resulted in the production of immunosuppressive cytokines such as TGF $\beta$ and in the resistance to TNF $\alpha$ or TRAIL-induced apoptosis.
There is also evidence that bacteria present in the tumor microenvironment are able to promote tumor growth via TLR signaling. Huang et al. [14] have shown that Listeria monocytogenes when present in the microenvironment of large, but not small tumors, accelerate tumor growth via TLR2 signaling. Apparently the same or similar mechanism operates in gastric carcinoma patients due to the interaction of their cells expressing TLRs with Helicobacter pylori bacteria, in contrast to normal situation, when interactions between commensal bacteria and gastro-intestinal mucosa through Toll-like receptors maintain homeostasis $[15,41]$. Similarly, human myeloma cells express several TLRs, such as TLR1, 7 and 9. Their activation by bacterial ligands from infecting bacteria promote tumor growth and the escape from conventional therapies [16].

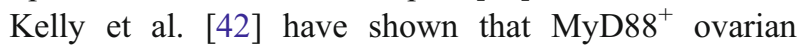
cancer cell line cells differ from those MyD88 ${ }^{-}$. LPS signaling of ${\mathrm{MyD} 88^{+}}^{+}$cancer cells resulted, apart from production of proinflammatory cytokines, in the induction of tumor growth. Moreover, MyD88 expression was associated with anti-cancer drug, paclitaxel resistance and expression of antiapoptotic proteins. It was not the case, when MyD88 $8^{-}$cancer cells were tested. This suggests, that MyD88 adaptor protein expression in cancer cells is of crucial importance in tumor biology.

Kundu et al. [17] have shown that immortalized prostate epithelial cells, expressing both TLR4 and TLR9, exhibit enhanced proliferation when cultured in the presence of lipopolysaccharide (LPS) and CpG DNA, their respective ligands. These stimulated cells were shown to be less susceptible to TNF-alpha induced apoptosis and to cell death due to high concentrations of LPS. Other authors such as Ilvesaro et al. [18] provided evidence that TLR9 agonistic unmethylated $\mathrm{CpG}$ oligonucleotides (CpG-ODN) promote matrix metalloproteinase-13 (MMP-13) activity resulting in enhanced migration of human prostate cancer cells expressing TLR9. Both LPS and CpG-ODN are wellknown surrogate molecules for pathogens residing in genitourinary system such as E. coli, and some DNA viruses (HPV for example). Overall, these data show that pathogens frequently encountered in this milieu may enhance malignant transformation and boost cancer cell spreading.

Moreover, it has been found that not only natural, but also synthetic CpG-ODN may function as vaccine adjuvants for infectious diseases as well as for cancer [19].

The role of TLRs expressed on tumor cells in the evasion of immune surveillance was elegantly demonstrated in animal experiments [20]. These authors have shown on several mouse tumor and tumor cell line systems that TLR4 activation by LPS enhances immune suppression in vitro. This inhibitory effect could be reversed by both, TLR4 short interfering RNA (siRNA) and TLR4 peptide. TLR4 stimu- 
lation of tumor cells resulted in synthesis of nitric oxide and several proinflammatory cytokines such as IL-6 and IL-12. Both, nitric oxide and IL-6 were shown to inhibit T cell proliferation and NK cell activity. Blockage of tumor TLR4 signaling by siRNA or TLR4 peptide led to prolongation of survival of tumor-bearing mice. These results show unequivocally that TLRs stimulation may lead to tumor progression and there are now means able to specifically reverse this unwanted effect.

On the other hand, Salaun et al. [21] demonstrated, that TLR3 on human breast cancer cells may drive these cells to apoptosis. Synthetic dsRNA, TLR3 agonist, induced apoptosis of 3 out 4 human breast cancer cell lines. Apoptosis was both, TLR3 and TRIF (adaptor molecule) dependent. TLR3 expressed by tumor cells was also able to drive cells into apoptosis, when stimulated by type I interferon. These data are important, because they provide evidence that cancerous cells may be triggered to death by endogenously derived factor. Functional TLRs expression has been demonstrated on cells of several human cancers, including, for example, squamous cell lung carcinoma cells expressing TLR9 [22].

We were able to show expression of TLR2, TLR3 and TLR4 on tumor cells of laryngeal carcinoma, using frozen tissue sections, appropriate antibodies, respective blocking peptides to assure specificity, and immunohistochemistry [23]. This tumor, in overwhelming majority originates from squamous, often keratinizing epithelium. Keratinocytes were shown to express functional Toll-like receptors [24]. It was also of interest from results of our study, that TLRs were found to be co-expressed with HLA-DR antigens, what might suggest the role of TLRs in putative tumor antigen presentation. Our recent data from experiments done on tissue culture cell lines of HNSCC hint for reverse biological function of TLR4 and TLR9. TLR4 expression seemed to support tumor progression, while TLR9 sensitized tumor cells to drug-mediated apoptosis [unpublished].

\section{TLR Agonists as Possible Agents in Tumor Immunotherapy}

The overall data presented above suggest that TLRs action in malignant process may be twofold: stimulation may promote tumor progression or it may have anti-tumor effect [27]. The latter action became recently the area of intensive study. It has been noticed by several investigators that some agonists of TLRs may induce strong anti-tumor activity. The best known such agent is imiquimod, a TLR7 agonist, used in the treatment of basal and squamous cell carcinoma [25, 26].

Okamoto and Sato [28] have isolated organic compound OK-432 and later its component OK-PSA from Streptococcus pyogenes strain. They have shown that these agents induce strong anti-cancer immunity via TL4/MD2 signaling in a mouse model. Moreover, they also reported that OK-432 based immunotherapy is effective in the treatment of patients with oral squamous cell carcinoma [29].

In the case of human melanoma, the malignancy subjected to the most extensive research programs, it was found, that human melanoma cells express functional TLR3 protein. TLR3 agonists can directly prevent cell proliferation and even induce cell death, when such treatment is combined with either type I interferon or inhibitors of protein synthesis [30].

In chronic lymphocytic leukemia tumor cells show strong expression of a large variety of TLRs, especially TLR7 and TLR9. Activation of the former by the respective agonists such as imidazoquinolines, TLR7 agonists may make tumor cells more sensitive to killing by both, cells of the immune system and chemotherapeutic agents. This is now a subject of phase I trials in several centers [31].

Schmidt et al. [32] have recently shown that also intratumoral injection of TLR2/6 agonist (macrophage activating lipopeptide-2, MALP-2) significantly prolonged survival of patients with pancreatic carcinoma, from 9 to 17 months. These findings are of importance, because they show interactions between TLRs and their agonists are

Table 1 Examples of clinical trials using TLR agonists in cancer patients

\begin{tabular}{|c|c|c|c|c|}
\hline Agent agonist & Cancer & Details & Results & Ref. \\
\hline $\begin{array}{l}\text { Imiquimod (TLR7 } \\
\text { agonist) }\end{array}$ & $\begin{array}{l}\text { Basal and squamous } \\
\text { cell carcinoma }\end{array}$ & $\begin{array}{l}\text { Topical application of } 5 \% \text { cream } \\
\text { five times weekly for } 6 \text { weeks }\end{array}$ & $\begin{array}{l}\text { Residual tumor in } 7.5 \% \text { of } \\
\text { patients }\end{array}$ & $\begin{array}{l}\text { Tillman and Carroll } \\
{[26]}\end{array}$ \\
\hline TLR 3 agonist & Melanoma & $\begin{array}{l}\text { Combined with IFN type I or } \\
\text { with protein synthesis inhibition }\end{array}$ & $\begin{array}{l}\text { Inhibition of cell proliferation } \\
\text { induction of cell death }\end{array}$ & Salaun et al. [30] \\
\hline $\begin{array}{l}\text { PF3512676 (TLR9 } \\
\text { agonist) }\end{array}$ & $\begin{array}{l}\text { Metastatic } \\
\text { melanoma }\end{array}$ & $6 \mathrm{mg} \mathrm{S.C./24} \mathrm{weeks}$ & Stimulation of innate response & Pashenkov et al. [33] \\
\hline $\begin{array}{l}\text { Imidazoquinoline } \\
528690 \text { (TLR7) }\end{array}$ & $\begin{array}{l}\text { Chronic } \\
\text { lymphocytic B } \\
\text { leukemia }\end{array}$ & TLR7 tolerization by agonist & $\begin{array}{l}\text { Increased sensitivity of tumor } \\
\text { cells to cytotoxic chemotherapeutics }\end{array}$ & $\begin{array}{l}\text { Spaner and Masellis } \\
\text { [31] }\end{array}$ \\
\hline $\begin{array}{l}\text { TLR2/6 MALP-2 } \\
\text { (synthetic) }\end{array}$ & $\begin{array}{l}\text { Pancreatic } \\
\text { carcinoma }\end{array}$ & $\begin{array}{l}\text { Intratumoral together with } \\
\text { gemcitabine }\end{array}$ & $\begin{array}{l}\text { Prolongation of life for } 8 \text { months } \\
\text { in average }\end{array}$ & Schmidt et al. [32] \\
\hline
\end{tabular}


effective locally, in tumor microenvironment. Examples of human tumor immunotherapy using TLR agonists are shown in Table 1. Current situation in this field, prospects and challenges have been recently discussed by Rezaei [34] and Romagne [35].

The question remains, what is the mechanism of beneficial effect of TLRs activation by agonists in malignant disease. Several lines of evidence indicate, that tumor cells exert inhibitory effect on various aspects of the immune system. It is manifested by the inhibition of maturation of dendritic cells [36], by the effect of secreted immunosuppressive cytokines such as IL-10 and TGF $\beta$, as shown in our previous studies [37] and other.

It appears that TLR-mediated signaling induced by agonists enhances the maturation of dendritic cells. It results in their migration to the regional lymph nodes, presentation of tumor antigens to $\mathrm{T}$ cells, production of cytokines such as IL-12 and IL-18, what all together redirects immune response to Th-1 type, beneficial for cancer patients in most clinical situations [28].

It remains of interest, how TLR signaling in malignant disease may be linked to the effects of conventional anti-cancer treatment such chemotherapy and radiotherapy. The data provided by French group, of Apetoh et al. [38] shed new light on this fascinating issue. These authors have found that dying tumor cells secrete peculiar alarmin protein, called high-mobility group box 1 (HMGB1) able to react with TLR4 on dendritic cells. This provides DC an impetus for efficient processing and cross-presentation of antigens from degraded tumor cells. Moreover, in the case of TLR4 polymorphism, manifested by TLR4 loss-of-function allele this DC function was lost, what resulted in early tumor relapse, at least in breast cancer patients [39]. It remains to find whether other cancer cells secrete such alarmin protein as breast cancer ones.

However, one should be aware of the fact, that not all tumor cells express TLRs, and not all TLR agonists as well as not all types of cell signaling mediated by TLRs induce "good" Th1-type response. Besides, TLR polymorphisms on tumor cells may produce unwanted effects during attempts of artificial TLR signaling by applied ligands [40]. Thus, the field of TLR-mediated tumor immunotherapy and links of TLRs signaling with conventional anti-tumor therapies still awaits intensive research efforts.

Acknowledgements The preparation of this review article was supported by two research grants from the Polish Ministry of Science and Higher Education: no. NN4011833 33 (to Prof. J. Żeromski) and no. N4011747 (to Dr. I. Mozer-Lisewska).

\section{References}

1. Vasselon T, Detmers PA (2002) Toll receptors: a central element in innate responses. Infect Immun 3:1033-1041
2. Lien E, Ingalls R (2002) Toll-like receptors. Crit Care Med 30:1-11

3. Akira S (2003) Mammalian toll-like receptors. Curr Opin Immunol 15:5-11

4. Banerjee A, Gerondakis S (2007) Coordinating TLR-activated signaling pathways in cells of the immune system. Immunol Cell Biol 85:420-424

5. Seitz M (2003) Toll-like receptors: sensors of the innate immune system. Allergy 58:1247-1249

6. Chen K, Huang J, Gong W et al (2007) Toll-like receptors in inflammation, infection and cancer. Int Immunopharmacol 7:1271-1285

7. Paterson HM, Murphy TJ, Purcell EJ et al (2003) Injury primes the innate immune system for enhanced Toll-like receptor reactivity. J Immunol 171:1473-1483

8. Okamura Y, Watari M, Jerud ES et al (2001) The extra domain A of fibronectin activates toll-like receptor 4. J Biol Chem 276:10229 10233

9. Beg A (2002) Endogenous ligands of Toll-like receptors: implications for regulating inflammatory and immune responses. Trends Immunol 23:509-512

10. Thomassen E, Renshaw BR, Sims JE (1999) Identification and characterization of SIGIRR, a molecule representing a novel subtype of the IL-1R superfamily. Cytokine 11:389-399

11. Lang T, Mansell A (2007) The negative regulation of Toll-like receptor and associated pathways. Immunol Cell Biol 85:425-434

12. Hassan F, Islam S, Tumurkhuu G et al (2006) Intracellular expression of toll-like receptor 4 in neuroblastoma cells and their unresponsiveness to lipopolysaccharide. BMC Cancer 6:281-289

13. He W, Liu Q, Wang L et al (2007) TLR4 signaling promotes immune escape of human lung cancer cells by inducing immunosuppressive cytokines and apoptosis resistance. Mol Immunol 44:2850-2859

14. Huang B, Zhao J, Shen S et al (2007) Listeria monocytogenes promotes tumor growth via tumor cell toll-like receptor 2 signaling. Cancer Res 67:4346-4352

15. Fukata M, Abreu MT (2008) Role of toll-like receptors in gastrointestinal malignancies. Oncogene 7:234-243

16. Jego G, Bataille R, Geffroy-Luseau A et al (2006) Pathogenassociated molecular patterns are growth and survival factors for human myeloma cells through toll-like receptors. Leukemia 20:1130-1137

17. Kundu SD, Lee C, Billips BK et al (2008) The toll-like receptor pathway: a novel mechanism of infection-induced carcinogenesis of prostate epithelial cells. Prostate 68:223-229

18. Ilvesaro JM, Merrell MA, Swain TM et al (2007) Toll-like receptor-9 agonists stimulate prostate cancer invasion in vitro. Prostate 67:774-781

19. Jurk M, Vollmer J (2007) Therapeutic applications of synthetic $\mathrm{CpG}$ oligodeoxynucleotides as TLR9 agonists for immune modulation. BioDrugs 21:387-401

20. Huang B, Zhao J, Li H et al (2005) Toll-like receptors on tumor cells facilitate evasion of immune surveillance. Cancer Res 65:5009-5014

21. Salaun B, Coste I, Rissoan MC et al (2006) TLR3 can directly trigger apoptosis in human cancer cells. J Immunol 176:4894-4901

22. Droemann D, Albrecht D, Gerdes J et al (2005) Human lung cancer cells express functionally active toll-like receptor 9 . Respir Res 6:1-10

23. Szczepański M, Stelmachowska M, Stryczyński Ł et al (2007) Assessment of expression of toll-like receptors 2, 3 and 4 in laryngeal carcinoma. Eur Arch Otorhinolaryngol 264:525-530

24. Pivarcsi A, Bodai L, Rethi B et al (2003) Expression and function of toll-like receptors 2 and 4 in human keratinocytes. Int Immunol $15: 721-730$

25. Urosevic M, Dummer R, Conrad C et al (2005) Disease independent skin recruitment and activation of plasmocytoid 
predendritic cells following imiquimod treatment. J Natl Cancer Inst 97:1143-1153

26. Tillman DK Jr, Caroll MT (2007) Topical imiquimod therapy for basal and squamous cell carcinomas: a clinical experience. Cutis 79:241-248

27. Killeen SD, Wang JH, Andrews EJ et al (2006) Exploitation of the toll-like receptor system in cancer: a doubled-edged sword? Br J Cancer 95:247-252

28. Okamoto M, Sato M (2003) Toll-like receptor signaling in anticancer immunity. J Med Investig 50:9-24

29. Sato M, Harada K, Yoshida H et al (1997) Therapy for oral squamous cell carcinoma by tegafur and streptococcal agent OK432 in combination with radiotherapy: association of the therapeutic effect with differentiation and apoptosis in the cancer cells. Apoptosis 2:227-238

30. Salaun B, Lebecque S, Matikainen S et al (2007) Toll-like receptor 3 expressed by melanoma cells as a target for therapy? Clin Cancer Res 13:4565-4574

31. Spaner DE, Masellis A (2007) Toll-like receptor agonists in the treatment of chronic lymphocytic leukemia. Leukemia 21:53-60

32. Schmidt J, Welsch T, Jäger D et al (2007) Intratumoural injection of the toll-like receptor-2/6 agonist' macrophage activating lipopeptide- $2 \varnothing$ in patients with pancreatic carcinoma: a phase I/II trial. Br J Cancer 97:598-604

33. Pashenkov M, Goëss G, Wagner C (2006) Phase II trial of a tolllike receptor 9-activating oligonucleotide in patients with metastatic melanoma. J Clin Oncol 24:5716-5724
34. Rezaei N (2006) Therapeutic targeting of pattern-recognition receptors. Int Immunopharmacol 6:863-869

35. Romagne F (2007) Current and future drugs targeting one class of innate immunity receptors: the toll-like receptors. Drug Discov Today 12:80-87

36. Idoyaga J, Moreno J, Bonifaz L (2007) Tumor cells prevent mouse dendritic cell maturation induced by TLR ligands. Cancer Immunol Immunother 56:1237-1250

37. Sikora J, Dworacki G, Kaczmarek M et al (2004) Immunosuppressive mechanisms in the microenvironment of malignant pleural effusions. Cancer Detect Prev 28:325-330

38. Apetoh L, Ghiringhelli F, Tesniere A et al (2007a) Toll-like receptor 4-dependent contribution of the immune system to anticancer chemotherapy and radiotherapy. Nat Med 13:10501059

39. Apetoh L, Ghiringhelli F, Tesniere A et al (2007b) The interaction between HMGB1 and TLR4 dictates the outcome of anticancer chemotherapy and radiotherapy. Immunol Rev 220:47-59

40. Tahara T, Arisawa T, Wang F et al (2007) Toll-like receptor 2 196 to 174del polymorphism influences the susceptibility of Japanese people to gastric cancer. Cancer Sci 98:1790-1794

41. Fukata M, Abreu MT (2007) TLR4 signaling in the intestine in health and disease. Biochem Soc Trans 35:1473-1478

42. Kelly MG, Alvero AB, Chen R et al (2006) TLR-4 signaling promotes tumor growth and paclitaxel chemoresistance in ovarian cancer. Cancer Res 66:3859-3868 